\title{
The effect of loading on surface roughness at the atomistic level
}

\author{
Peter Spijker • Guillaume Anciaux • \\ Jean-François Molinari
}

Received: 15 October 2010 / Accepted: 13 January 2011 / Published online: 4 February 2011

(c) Springer-Verlag 2011

\begin{abstract}
One of the key points to better understand the origins of friction is to know how two surfaces in contact adhere to one another. In this paper we present molecular dynamics (MD) simulations of two aluminium bodies in contact, exposed to a range of normal loads. The contact surfaces of both aluminium bodies have a self-affine fractal roughness, but the exact roughness varies from simulation to simulation. Both bodies are allowed to have an adhesive interaction and are fully deformable. Tracking important contact parameters (such as contact area, number of contact clusters, and contact pressure) during a simulation is challenging. We propose an algorithm (embedded within a parallel MD code) which is capable of accessing these contact statistics. As expected, our results show that contact area is increasing in proportion with applied load, and that a higher roughness reduces contact area. Contact pressure distributions are compared to theoretical models, and we show that they are shifted into the tensile regime due to the inclusion of adhesion in our model.
\end{abstract}

Keywords Contact mechanics - Molecular dynamics . Friction · Roughness · Nanotribology

\section{Introduction}

To understand friction has been the goal for many researchers from the ancient Greeks until the current day. Although a lot is known about the factors contributing to friction, a complete picture of the origins of friction is still lacking. At the macroscopic level it is understood since the days of Da Vinci,

P. Spijker $(\varangle) \cdot$ G. Anciaux · J.-F. Molinari

Computational Solid Mechanics Laboratory,

Ecole Polytechnique Fédérale de Lausanne (EPFL),

Lausanne, Switzerland

e-mail: peter.spijker@epfl.ch
Amontons and Coulomb that the force of friction is directly proportional to the applied load and that the apparent contact area does not influence the frictional force $[2,11]$. The direct proportionality between the applied load $F_{N}$ and the frictional force $F$ yields a dimensionless constant $\mu$ known as the friction coefficient.

One of the important topics in current tribology research is the influence of surface roughness on friction [5]. It has long been shown that a simplistic geometrical representation of surface roughness is not sufficient, and that real surfaces have a quite different type of roughness, with asperities at different length scales, which can be approximated by selfaffine fractals [21,31]. As a consequence the real contact area $A_{R}$ of two materials is much smaller than the apparent contact area $A$, because the materials only touch at a few high spots (asperities) of both respective surface landscapes, see Fig. 1.

Bowden and Tabor proposed a model for friction in which the tangential force is proportional to the real contact area and to the shear strength of each contacting asperity (e.g., assuming the occurrence of plastic deformation) [6]. They also investigated friction for a purely elastic sliding process, and based on the Hertz theory of elastic contacts, found a contradicting non-linear dependence of friction on load: $F \propto F_{N}^{2 / 3}[6,26]$. This contradiction was resolved by Archard [4] by assuming that contact involves many asperities and that the number of contacting asperities is load dependent. Greenwood and Williamson [13] improved Archard's method even further by assuming Gaussian and exponential height distributions for the asperities. One of the drawbacks of the Greenwood-Williamson approach is that it relies on a known height distribution, and that it does not take into account the elastic coupling between asperities. In later work many of these limitations on the Greenwood-Williamson theory have been removed $[9,30]$. In order to cope with 


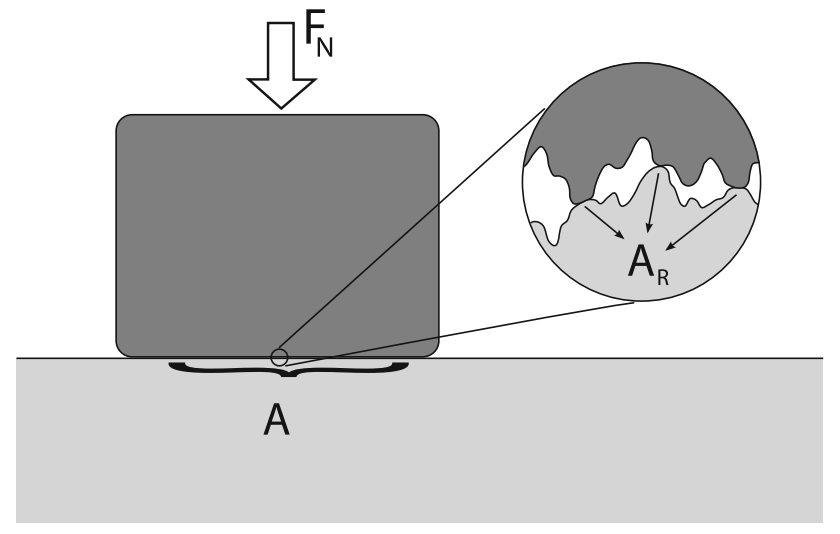

Fig. 1 A schematic depiction of two bodies in contact due to a normal force $\left(F_{N}\right)$. When zooming in on the contact interface it is observed that the real area of contact $\left(A_{R}\right)$ is much smaller than the apparent area of contact $(A)$, because the two bodies only contact on a small number of asperities due to surface roughness

different length scales of asperities at once, Persson [31] proposed a theory which is based on the auto-correlation of the surface heights.

Most of the approaches mentioned above describe the surface from a continuum mechanics point of view. However, in the limit of smaller length scales contact occurs finally at the atomistic level, where the continuum theory may become inadequate $[18,19]$. To better understand the origins of friction it is important to analyze how two surfaces adhere to each other at the atomistic level. In this paper we use molecular dynamics (MD) simulations to investigate two aluminium bodies in contact due to normal loading. Previous similar MD simulations mainly covered two flat crystal surfaces in contact, either commensurate or amorphous $[3,19]$. In this work our interest lies with rough crystal surfaces in contact. Some work has been done on rough surfaces, but mainly in 2D [14,20], or in 3D with one of the bodies rigid [7]. In this paper we aim at a full 3D description of the problem with both bodies deformable, which to the best of our knowledge is done here for the first time.

We investigate a range of loading pressures and different types of atomistic scale roughness. Determining the parameters that are of importance to the contact problem (such as contact area and pressure) is a challenging task, and especially if this needs to be done on the fly. We present the foundations of an algorithm that can be used within a MD simulation to measure these contact parameters in real time. This algorithm is implemented within the parallel MD code LAMMPS [32].

In the following sections we describe the model setup and validation including the explanation of the new contact detection algorithm. In the sections thereafter we present and discuss the results of the MD simulations. The final section presents a summary and conclusion.

\section{Methods}

\subsection{Interaction potential}

Molecular dynamics simulations are an interesting tool to investigate physical processes at the level of individual atoms. At its core this technique solves Newton's equations of motion for each individual atom in the system under consideration. In order to do so, interactions between atoms need to be accounted for, for instance by using pair-wise potential energy functions between the atoms. A well-known example of such pair-wise potential is the Lennard-Jones (LJ) potential [17]

$V_{\mathrm{LJ}}(r)=4 \epsilon\left[\left(\frac{\sigma}{r}\right)^{12}-\left(\frac{\sigma}{r}\right)^{6}\right]$

where $r$ is the distance between two atoms, $\sigma$ their characteristic length, and $\epsilon$ the energy at which the potential is at its minimum. Although designed for MD simulations of noble gases [1], the LJ potential is also used successfully in biological MD simulations $[15,16,23,33]$ as well as in computational mechanics $[8,19,34]$. Other potentials can be used to describe the interactions between atoms, such as the Morse potential or the embedded atom method [12,27], but for the system under investigation in this work the LJ potential suffices.

The material that is modeled in all simulations is aluminium, because its crystal structure is face centered cubic (FCC), which is naturally recovered by the LJ potential. To model the properties of aluminium, it is required to get correct LJ parameters $\sigma$ and $\epsilon$. Given the density of aluminium (2.70 $\left.\mathrm{g} / \mathrm{cm}^{3}\right)$ and its mass $(26.98 \mathrm{~g} / \mathrm{mol})$ the crystal lattice constant can be determined $(0.404 \mathrm{~nm})$. The simulations should start from a crystal in a stable configuration, and, for this purpose we use the configuration of minimal energy to determine the characteristic length $\sigma$. Taking into account all nearest neighbors up to the fourth layer in the crystal, the characteristic length for aluminium equals $\sigma=0.2596 \mathrm{~nm}$. Using the minimum energy configuration to compute $\sigma$ ensures that the crystal does not contract or expand in the bulk regions.

Modeling some mechanical parameters of aluminium correctly with the $\mathrm{LJ}$ model is important. Therefore, we derived the energy parameter $\epsilon$ by fitting it to aluminium's Young's modulus of $68 \mathrm{GPa}$. Assuming the crystal is infinitely large and using the Cauchy-Born rule at zero Kelvin [35], we can proceed to determine the full elastic tensor, which is given by

$\mathbf{C}=\frac{1}{2 \Omega} \sum_{i}\left[\frac{1}{r_{i}^{2}} \frac{\partial^{2} V_{\mathrm{LJ}}}{\partial r_{i}^{2}}-\frac{1}{r_{i}^{3}} \frac{\partial V_{\mathrm{LJ}}}{\partial r_{i}}\right] \mathbf{r}_{\mathbf{i}}^{\mathbf{0}^{\otimes 4}}$,

where $\mathbf{C}$ is the elastic tensor, $\Omega$ the volume associated with any atom, $r_{i}$ the distance from the arbitrary center of 
the crystal, $\mathbf{r}_{i}^{0}$ the undeformed positions of the atoms, $V_{\mathrm{LJ}}$ the interaction potential (in this case the LJ-potential), and $\mathbf{r}_{\mathbf{i}}^{\mathbf{0}} \otimes 4=\mathbf{r}_{\mathbf{i}}^{\mathbf{0}} \otimes \mathbf{r}_{\mathbf{i}}^{\mathbf{0}} \otimes \mathbf{r}_{\mathbf{i}}^{\mathbf{0}} \otimes \mathbf{r}_{\mathbf{i}}^{\mathbf{0}}$. As an approximation to isotropic material the Poisson ratio and Young's modulus can be taken as

$\nu=\frac{C_{11}-C_{12}}{2 C_{11}-C_{12}} \quad$ and $\quad E=C_{11}(1+v)$.

It can be easily seen that the Young's modulus depends only on the energy parameter, and that there even exists a linear relationship between the two. Based on aluminium's Young's modulus this gives for the LJ energy parameter $\epsilon=10.3014 \mathrm{~kJ} / \mathrm{mol}$.

The cut-off radius for the $\mathrm{LJ}$ potential is set at $0.6039 \mathrm{~nm}$ (at which point the potential is almost zero) and for searching the nearest neighbor list an extra shell with a width of $0.234 \mathrm{~nm}$ is added. To avoid discontinuities in the Van der Waals energy the potential is shifted to make sure that at the cut-off radius the potential energy equals zero. The values used in the simulations for the interaction energy between the two contacting bodies are discussed in a subsequent section.

We performed MD simulations of simple tensile tests in different lattice directions. When the loading is in the principal direction (100) we recovered the Young's modulus for aluminium used as an input, but performing the same test in the direction perpendicular to the packed plane (111) gives a higher Young's modulus, see Fig. 2. Thus, our simple aluminium LJ model has a degree of anisotropy, which is slightly higher that what is observed in experiments [29]. However, in our simulations we apply all the loading along the principal directions (where we recover the correct Young's modulus),

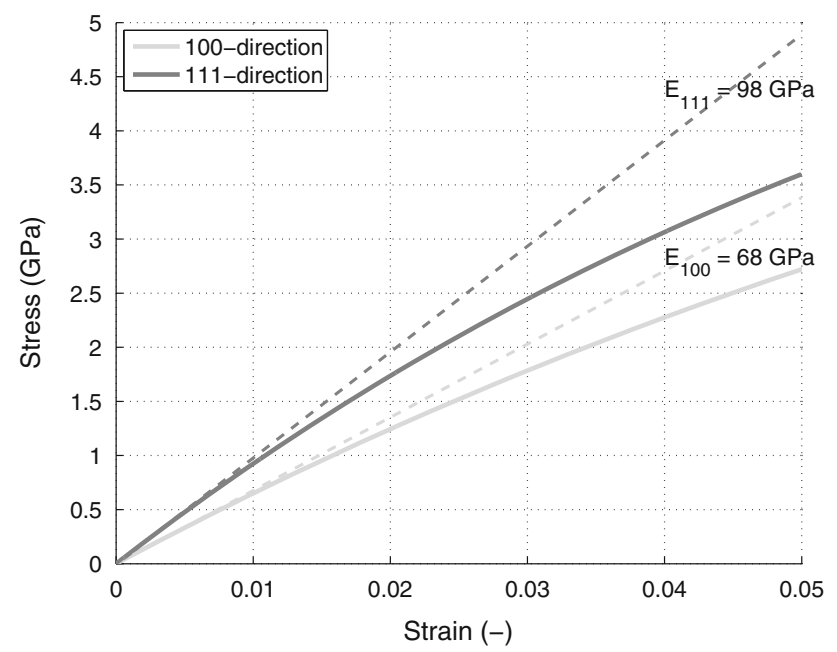

Fig. 2 The resulting stress-strain curve from the tensile test MD simulations with the determined LJ parameters. The response differs depending on the loading direction (along the principal directions, 100, or perpendicular to the packed plane, 111). The tangents at zero stress and strain (dashed lines) are used to compute the Young's modulus as shown in the figure giving us confidence that our LJ model describes aluminium well enough for our current purposes.

\subsection{Rough surface generation}

Many surfaces have some sort of roughness on all length scales, including the atomistic level, that can be described by a self-affine fractal scaling, with the Hurst exponent $H$ playing a pivotal role in quantifying the roughness [3,14,31]. Other interesting quantities that characterize the rough surface are the root mean square (RMS) roughness, the maximum peak to minimum valley distance, the height skewness and kurtosis, and the RMS slope. Using the random midpoint displacement (RMD) algorithm [24,36], which has the Hurst exponent as one of its inputs, a rough surface can be generated which is periodic across its boundaries. The original RMD algorithm starts by displacing the center of the underlying square surface grid, and in the next step taking the four points between the center and the corners, and so on until the algorithm arrives at the smallest possible dimension of the grid. However, by using the RMD algorithm in this way it is likely that the generated surface has only a few, albeit large asperities. This can be unfavorable if one wants a larger number of asperities, while still maintaining the same RMS roughness. To avoid this, the RMD algorithm should not start by displacing only the center, but by displacing the centers which would have emerged at a later stage in the algorithm instead. In other words, the algorithm does not start with one large square, but with multiple smaller but equally sized squares (e.g., 4 or 16). This is similar to moving the cutoff wave vector of the surface roughness power spectrum to higher frequencies [31]. To create a surface with a predefined RMS roughness, it is sufficient to scale the heights of the surface accordingly after it has been generated by the RMD algorithm.

\subsection{System setup}

One of the major drawbacks of MD is that its computational cost increases rapidly with the number of particles. As we wish to study different types of rough surfaces, we choose a relatively small system size in order to keep the computational cost acceptable. The aluminium crystal structures we create measure $32 \times 32$ unit cells in the lateral direction, which is equivalent to lateral dimensions of approximately $13 \times 13 \mathrm{~nm}$. To avoid interlocking of the two crystals when they are pressed together (due to the same crystal orientation) the underlying crystal structure of the upper body is rotated by $21^{\circ}$.

Because the behavior of the material is not known before and possible plasticity (including atom shuffling and the emission of dislocations) might occur when the two contacting bodies are loaded and pressed together, it is 
important to have sufficient bulk material [39]. Therefore, we choose to make each of the bodies cubic, giving them a depth of $13 \mathrm{~nm}$ as well.

After creating two crystals for either the upper or lower body of the system, we generated two rough surfaces. These surfaces were projected onto the crystals, and we removed any atoms above (lower body) or below (upper body) the corresponding rough surface, creating an atomistic equivalent of our rough aluminium sample. Consequently the cubic shape of either of the two bodies is slightly altered, but it is believed the remaining bulk material is sufficiently large to still resemble correctly mechanical properties.

In Fig. 3 a schematic depiction of the system setup is shown. Each of the two bodies (with its rough surface) is shown as well as the subdivision into different regions. For the lower body the lowest region (number 6) is kept fixed at all time, giving the system its support. On the contrary the highest region of the upper body (number 5) is kept rigid, but is allowed to move as one entity upon an externally applied pressure. The two main regions (numbers 1 and 2) are completely free (thus deformable), whereas two small thermostat regions (numbers 3 and 4 ) are used to maintain the

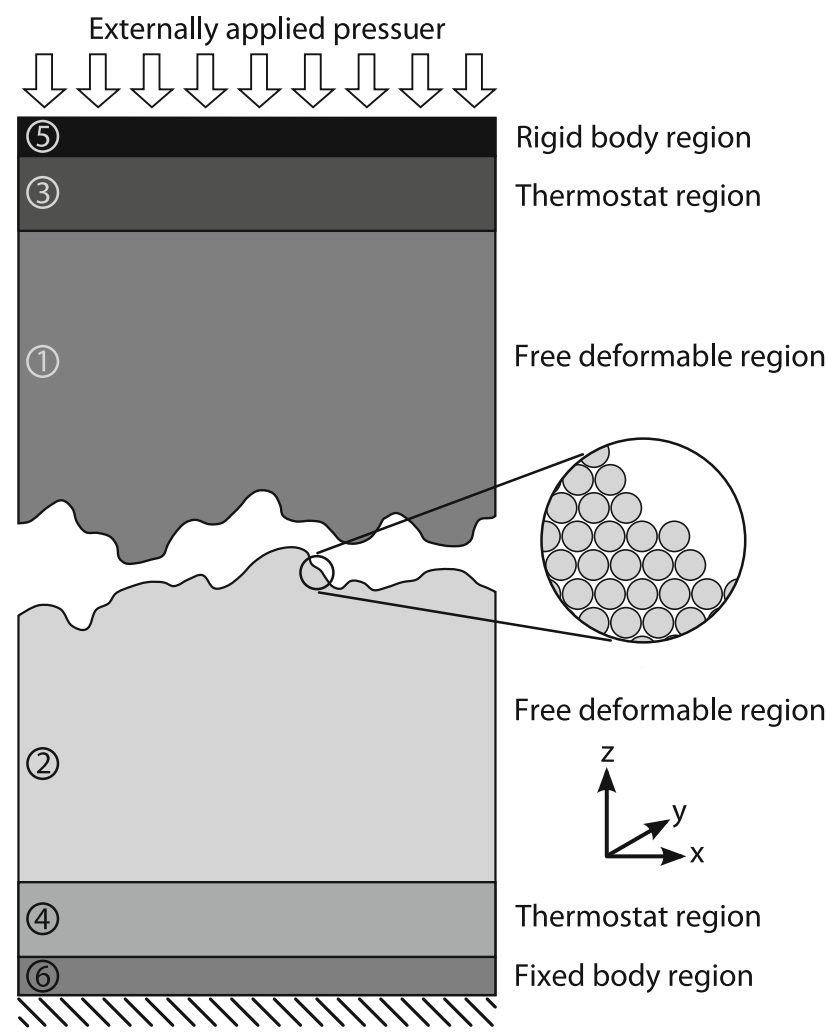

Fig. 3 Schematic representation of the system setup. The gray scales and numbers indicate different regions, where the upper domain is numbered with even numbers and the lower domain with odd numbers. The inset shows that the system has an atomistic representation. Though this schematic representation is $2 \mathrm{D}$ the system extends into the third dimension as well temperature in the system as constant as possible, but where the atoms are (except for their thermocoupling) free to move as well. Furthermore, in the $x$ - and $y$-directions the system is considered to be periodic. The size in $z$-direction of regions 5 and 6 (in terms of number of lattice layers) is 4 , for regions 3 and 4 this size is 8 , and for the main regions 1 and 2 approximately 40 layers.

It is important to note that although six regions are present in the system, each atom in any region is modeled to be aluminium. The benefit for naming the regions differently is to simplify both the simulations and the analysis.

\subsection{Simulations}

In order to investigate different roughnesses and different loading pressures a large number of MD simulations has to be performed. We have chosen to investigate three different surface roughness (with RMS roughness of $0.2,0.5$ and $1.0 \mathrm{~nm}$, respectively), and use three different random seeds to generate three surfaces for each roughness. Thus, in total we look at nine different surfaces. However, to avoid making the problem too complex, we have chosen to use only one Hurst exponent for our surfaces, namely 0.8. The same RMS roughness and Hurst exponent are used to generate the rough surfaces of the upper and lower bodies. In Fig. 4 the top view of the rough surfaces for the lower bodies for three different RMS roughnesses are shown as an example.

Although the RMS roughness is an input in the generation of the rough surfaces, due to the removal of atoms above or below the rough surface (depending on whether it is the upper or lower body), the actual RMS roughness of the atomistic surface can be changed to a small extent. To show this effect and also that all generated surfaces are quite different in their topology, we computed some statistically relevant parameters of the surface roughness, which are shown in Table 1.

The general observation from Table 1 is that skewness (asymmetry) and kurtosis (peakedness) are not influenced significantly by different RMS roughness and that the RMS slope is proportional to the RMS roughness. Furthermore, from this table it is observed that the RMS roughness of the atomistic representation is slightly less than the RMS roughness used as an input. This effect is caused by the fact that the atoms reside on a crystal lattice and the surface is thus discretized. The lattice discreteness can be observed from the peak to valley distances (column denoted by PV in the table), which are sometimes the same for different rough surfaces. Because the distance between subsequent atomistic crystal layers is fixed, the peak to valley difference is determined by the number of atomistic layers between the highest and lowest value of the atomistic surface.

To investigate the effect of Van der Waals interactions at the atomistic scale we have chosen to use two different LJ energy parameters for the interaction between the two 


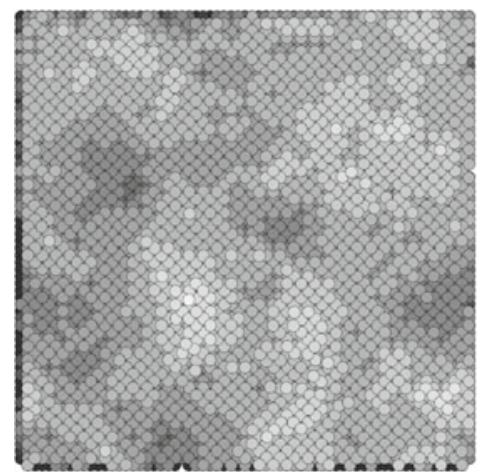

(a) $\operatorname{RMS} 0.2 \mathrm{~nm}$

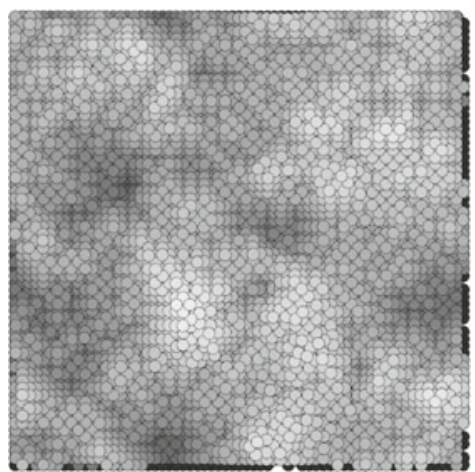

(b) RMS0.5nm

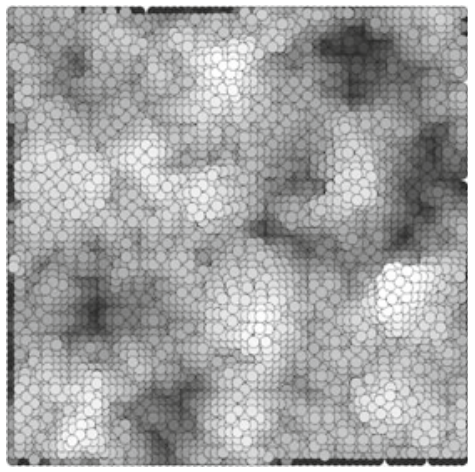

(c) RMS1.0nm

Fig. 4 Top views of the atomistic representation of the bottom surfaces for three systems with different RMS roughness (increasing from the left to the right figure). The darker or lighter the scale of grays, the lower or higher the valleys or peaks

Table 1 Surface statistics for the systems with different surface roughness used in the MD simulations

\begin{tabular}{|c|c|c|c|c|c|c|}
\hline $\mathrm{RMS}_{0}(\mathrm{~nm})$ & Random seed & $\mathrm{PV}(\mathrm{nm})$ & RMS (nm) & Skew. & Kurt. & RMS slope \\
\hline \multicolumn{7}{|c|}{ Upper body rough surface } \\
\hline 0.2 & 1 & 1.21 & 0.194 & 0.090 & 0.085 & 0.132 \\
\hline 0.2 & 2 & 1.01 & 0.196 & 0.145 & -0.076 & 0.127 \\
\hline 0.2 & 3 & 1.01 & 0.190 & 0.032 & -0.374 & 0.141 \\
\hline 0.5 & 1 & 2.82 & 0.466 & 0.046 & -0.069 & 0.300 \\
\hline 0.5 & 2 & 2.42 & 0.463 & 0.041 & -0.183 & 0.283 \\
\hline 0.5 & 3 & 2.42 & 0.454 & 0.056 & -0.609 & 0.323 \\
\hline 1.0 & 1 & 5.24 & 0.924 & 0.064 & -0.166 & 0.582 \\
\hline 1.0 & 2 & 5.24 & 0.910 & -0.139 & -0.120 & 0.579 \\
\hline 1.0 & 3 & 4.64 & 0.900 & 0.087 & -0.526 & 0.636 \\
\hline \multicolumn{7}{|c|}{ Lower body rough surface } \\
\hline 0.2 & 1 & 1.21 & 0.198 & -0.112 & 0.164 & 0.149 \\
\hline 0.2 & 2 & 1.21 & 0.192 & 0.018 & 0.112 & 0.133 \\
\hline 0.2 & 3 & 1.01 & 0.188 & -0.101 & -0.206 & 0.138 \\
\hline 0.5 & 1 & 2.82 & 0.461 & -0.217 & 0.130 & 0.332 \\
\hline 0.5 & 2 & 2.42 & 0.471 & 0.111 & 0.051 & 0.311 \\
\hline 0.5 & 3 & 2.42 & 0.452 & -0.103 & -0.425 & 0.328 \\
\hline 1.0 & 1 & 5.24 & 0.934 & -0.157 & 0.158 & 0.670 \\
\hline 1.0 & 2 & 6.05 & 0.958 & 0.026 & 0.353 & 0.617 \\
\hline 1.0 & 3 & 4.84 & 0.910 & -0.133 & -0.408 & 0.656 \\
\hline
\end{tabular}

The first table is for the rough surface of the upper body and the second for the rough surface of the lower body. The first column gives the RMS roughness $\left(\mathrm{RMS}_{0}\right)$ used as an input. All other columns give computed values. Differences between $\mathrm{RMS}_{0}$ and RMS show the effect of lattice discreteness. PV stands for peak to valley distance

bodies. To model the case of full adhesion between the two bodies $\epsilon_{1-2}$ is kept at the value for the bulk material $\left(\epsilon_{1-2}=\right.$ $10.3014 \mathrm{~kJ} / \mathrm{mol})$, and for the case where adhesion is almost completely absent $\epsilon_{1-2}$ is put at $10 \%$ of the bulk value $\left(\epsilon_{1-2}=\right.$ $1.03014 \mathrm{~kJ} / \mathrm{mol})$. The lack of adhesion can be seen as the effect of, for instance, an oxide layer on the metal, which reduces the adhesive energy considerably, but without modeling this oxide layer explicitly.

Furthermore, each of the nine different systems is subject to five different normal loads in the range of $0.05-0.25 \mathrm{GPa}$, each load being far below the yield strength of aluminium. Together with the change of the LJ energy parameter this puts the total number of MD simulations for the current work at 90. For all simulations the Large-scale atomic/molecular massively parallel simulator (LAMMPS) is used [32], with some important changes to the code to accommodate our analysis (see next section).

For each simulation the temperature of the entire system is set at $10 \mathrm{~K}$, modeling a situation as close as possible to $0 \mathrm{~K}$ without causing numerical instabilities. During the simulation this temperature is controlled in the thermostat regions through the use of a Langevin thermostat with the damping constant $250 \mathrm{fs}^{-1}$. Hence, there can be a heat flow in the bulk material from or to the thermostat regions. The integration time step for all simulations is set at $5 \mathrm{fs}$, and periodic boundary conditions are applied only in the lateral directions. 


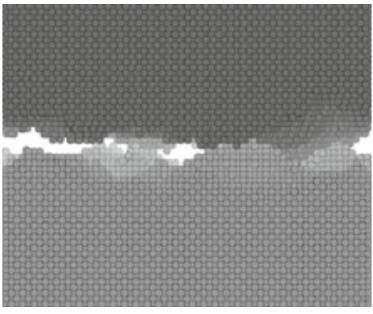

(a) Orthogonal view (b) Perspective view

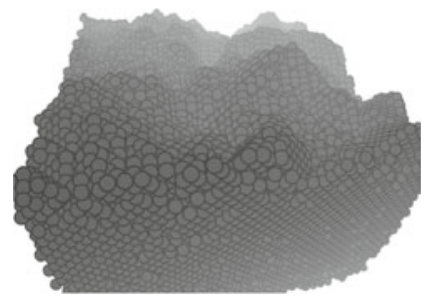

Fig. 5 Orthogonal side view (a) and perspective view (b) of the same system at the end of the initial loading process. The surface roughness equals $0.5 \mathrm{~nm}$ and the applied pressure is $0.10 \mathrm{GPa}$. In $\mathbf{b}$ the top domain is omitted to allow a good view of the rough surface

For each generated system, we make sure that the two bodies are not in contact, i.e., no atomic pair between two opposing surfaces is within the cut-off radius of the LJ potential. During the first part of each simulation the upper body is lowered quickly, and moves as a rigid body, until one of the atoms enters the neighborhood of an atom of the lower body. From this point on the lowest pressure $(0.05 \mathrm{GPa})$ is imposed on the topmost layer of atoms (region 5) and the upper body is allowed to descend gradually, contacting the lower body, until the entire system is in equilibrium (which takes about $250 \mathrm{ps}$ ). Thereafter the pressure is repeatedly increased by $0.05 \mathrm{GPa}$ and equilibrated again for $250 \mathrm{ps,}$ until the final equilibrated pressure of $0.25 \mathrm{GPa}$ is reached. In Fig. 5 an example of a loaded system is shown. For the equilibrated systems, at $0.05,0.10,0.15,0.20$ and $0.25 \mathrm{GPa}$, a snapshot of the system is obtained, which is used for any of the production runs. For these production runs a MD simulation with constant pressure on the rigid region of the upper body (region 5) is carried out for $1 \mathrm{~ns}$, where the last $500 \mathrm{ps}$ are used for analysis. A typical system for a MD simulation contains about 217,000 atoms and one production run takes about $7 \mathrm{~h}$ on eight processors of a Linux commodity cluster.

\subsection{Contact detection algorithm}

To monitor the evolution of loading, it is important to know which atoms of both separate bodies are in contact and at which time. However, when dealing with interactions due to classical potentials it is not so clear to determine when two atoms are in contact or not. Indeed atoms feel each other's presence from a distance of about $0.6 \mathrm{~nm}$, but at that distance exert only a small force on each other. For the sake of simplicity we have chosen to assume that two aluminium atoms are in contact when they are closer than $0.5 \mathrm{~nm}$, which implies that typically the first and second neighbors of an atom are assumed to be in contact with it.

As mentioned in the introduction, we have extended the LAMMPS code to compute this contact on the fly during the simulation. It has to be mentioned that most of this algorithm is developed with the aim of performing MD simulations of two bodies sliding on top of each other. Although the current work (normal loading) does not benefit from all possibilities the algorithm provides, we describe the entire algorithm for completeness.

At predefined time steps (for this work every 1,250 fs, thus 400 times during the analysis part of the production run) the system is analyzed and possible contact is examined. At each such time step we compute the current total force on each of the regions (decomposed in Cartesian directions), the contact area (projected on the $x y$-plane), the number and size of contacting clusters (also projected on the $x y$-plane), the force on each atom in contact due to atoms from its own body and from the other body, and the pressure contribution for each atom in contact. It is crucial for the algorithm that we can identify to which region an atom belongs, so we can compute the correct contact properties accordingly.

In order to evaluate the total force on each of the regions, the LAMMPS code has been changed in the innermost force computation loop. Whenever a force between the two atoms of the pair interaction is computed, the force is also stored but then as a force between two regions to which both atoms belong. In the current work this means a $6 \times 6 \times 3$ array of forces is computed for each time step (six regions and three Cartesian dimensions). Although the force decomposition in the case of normal loading only serves to see if the system is in equilibrium, it can be very useful in a sliding simulation, because it then easily allows for the computation of the friction coefficient.

The computation of the contact area is slightly more complicated, mainly because we wish to be able to distinguish individual contacting clusters. Moreover, if due to the loading, parts of one region break off and stick to the other region (thus, without being in contact with its original region), we do not want to count these atoms as being in contact, as they are no longer load bearing. That this might happen is not so evident in the case of normal loading, but, again, becomes important with sliding (where scratching of the surface might occur).

Therefore, all atoms that are in regions 1 and 2 are being sorted into clusters by a depth-first search (a black-white-gray cluster algorithm) [10], implemented in a parallel environment and taking the periodic boundary conditions in mind. Whenever a cluster is considered to be an orphan (i.e., the cluster is not connected to the bulk part of the region it should belong to, but instead connected to the other), the region number of the atoms in this cluster is changed to the number of their new parent region. Subsequently, these atoms are discarded as being in contact. By treating the atoms in this way, we ensure only considering atoms that are truly in contact, and, thus, that are load bearing.

After having determined the atoms that are in contact, we project them on a square grid lying in the $x y$-plane, where 


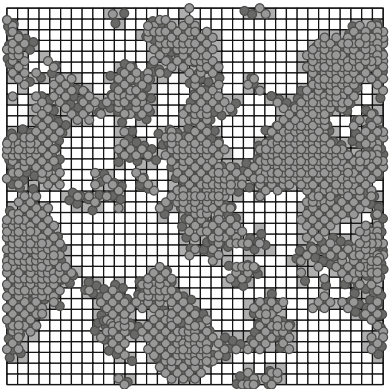

(a) With atoms

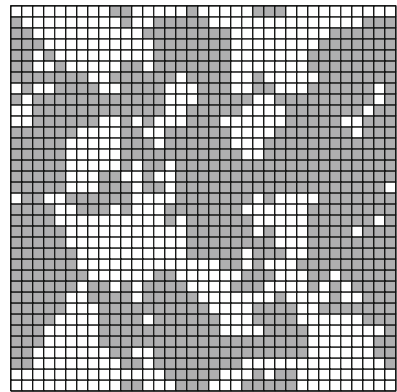

(b) Without atoms
Fig. 6 In a the atoms of both bodies that are in contact (atoms in lighter gray belong to lower body) are shown as projected on the $x y$-plane. The underlying grid used to determine the contact area is shown as well, and in $\mathbf{b}$ the grid representation of the contact area is shown (gray boxes). The contact area equals 0.58 (as fraction of the apparent contact area), when the RMS roughness is $0.2 \mathrm{~nm}$, the load is $0.15 \mathrm{GPa}$ and full adhesion between the two bodies is used

each grid cell measures 2.5 times the Van der Waals radius of an aluminium atom. By counting which grid cells are occupied by at least one atom, we can get a good estimate of the projected contact area. As an example of this computation, we show the projected contact area for one of the three cases with imposed RMS roughness of $0.2 \mathrm{~nm}$ and a $0.15 \mathrm{GPa}$ load in Fig. 6. Although the grid projection could result in a slight overestimate of the true contact area, this method has the advantage to allow for unambiguous contact area detection. Moreover, it easily allows determining the fraction of real and apparent contact area.

The next step in the algorithm is to determine the number of contacting clusters. This is done by using the same depthfirst search to sort the projected area grid into clusters (e.g., the example of Fig. 6 gives three clusters). At all time it is tracked to which cluster the individual atoms belong.

At the level of the atoms, the force on each atom is recorded and split whether it stems from the same or another region. Also, for each contacting atom its pressure contribution (computed using the virial theorem) is recorded.

By using the information on the contacting clusters and the individual atom information it is possible to compute the local pressure on each contact cluster.

Finally, to investigate the changes in the surface properties, the roughness statistics as presented in Table 1 are recomputed every $12.5 \mathrm{ps}$.

\section{Results}

We begin by considering the dependence of the contact area on applied load, surface roughness, and adhesive properties, which is shown in Fig. 7. In this figure the different surface roughnesses and adhesive properties (weak or full adhesion) are indicated by the different gray scales and markers.

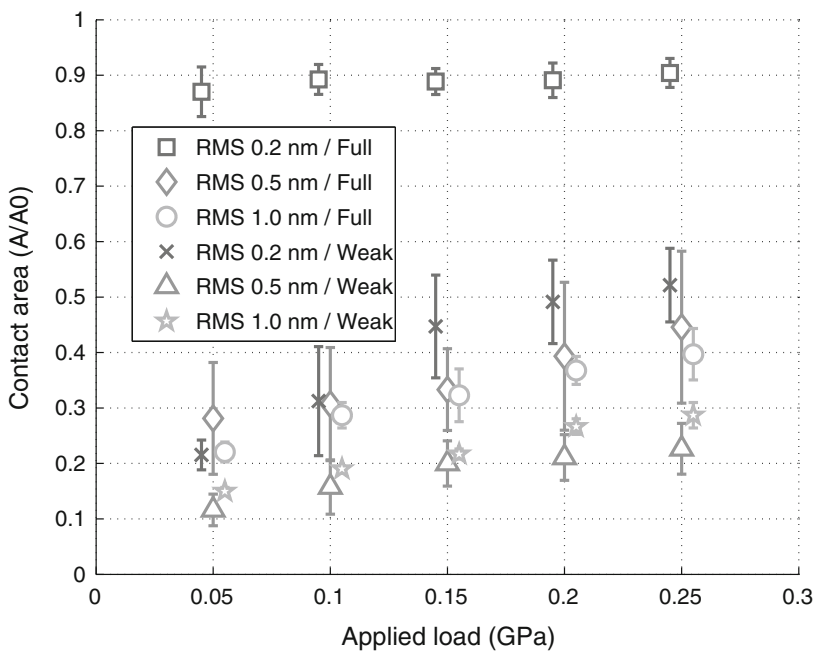

Fig. 7 The contact area as a fraction of the apparent area is shown for different loads, different surface roughness, and full and weak adhesion. Error bars indicate the spread due to the different random seeds used in the generation of the rough surfaces. The minor $x$-offsets for each pressure are introduced for clarity

The error bars indicate the standard deviation in the contact area due to the different random seeds used in the rough surface generation.

As expected, for all cases the contact area increases with increasing load $[7,14,19]$. This increase seems to be linear, but because we have used only five different loads, it is difficult to put the linearity into numbers. Furthermore, the error bars increase with decreasing RMS roughness, indicating that for the current sample size the topography of the surfaces is important. For the higher RMS roughness the topographies look more alike (more prominent asperities), whereas the differences for the lower roughnesses could lead to quite different surfaces being in contact. Note however that the RMS $0.2 \mathrm{~nm}$ full adhesion case is special. There, the system is almost in full contact for all applied loads, which leads to smaller error bars.

The difference between the adhesive properties is most clearly shown by the fact that the contact areas for the full adhesive cases are always larger than with weak adhesion. This is easily understood because with strong adhesion surfaces are attracted to each other and, thus, are likely to be in closer contact.

Carefully examining the results shows that the contact area also decreases with increasing RMS roughness, irrespective of the adhesive strength, although it remains more or less the same for a RMS roughness of $0.5 \mathrm{~nm}$ or higher. However, for the weak adhesion case the results for the two higher RMS roughnesses are reversed. This is most likely due to the relative small system size of our simulations, which gives a very small number of contacting asperities for higher RMS roughness. 


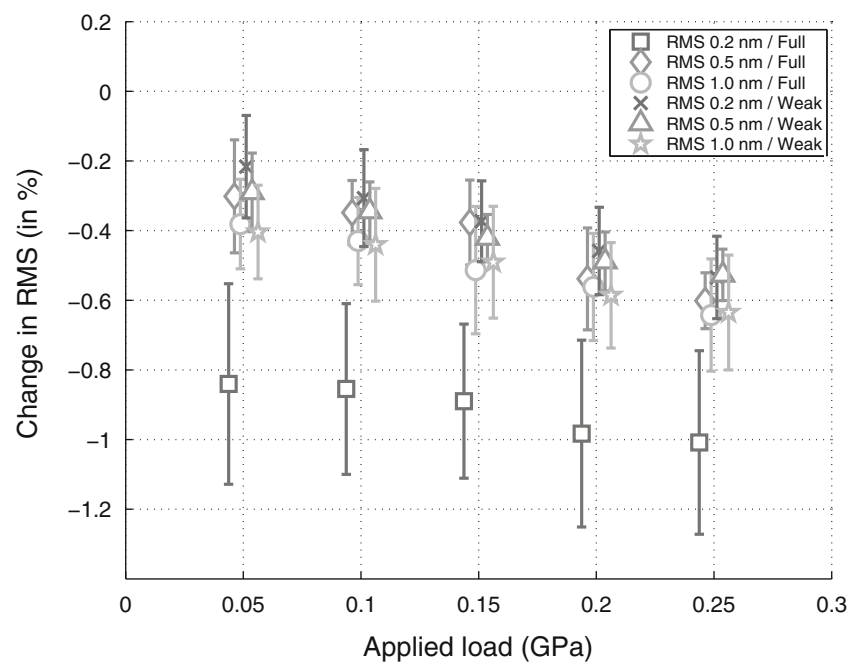

(a) RMS roughness

Fig. 8 Change of surface properties due to normal loading, either with full or weak adhesion. Shown are in a the relative change of RMS roughness and $\mathbf{b}$ the relative change of the RMS slope. Different gray scales

Due to the large loads imposed on the system it is expected that both surfaces change their roughness characteristics, see Fig. 8. In (a) the relative change of the RMS roughness is shown and in (b) the relative change of the RMS slope. The rough surfaces of both contacting and deformable bodies are used for this analysis, and to compute the relative change the values for the characteristics as found in Table 1 are used as a reference.

From Fig. 8a it can be seen that the RMS roughness changes (as a function of applied load), although to a very small extent (just over $1 \%$ in the most extreme case). The changes in the RMS slope, see Fig. 8b, are much more pronounced, and can go up to $10 \%$. Not shown in a figure is the change in skewness (a measure for the change in symmetry of the height distribution), but for all cases is on average $0.156 \pm 0.053$, which is almost without exception at least a doubling of the initial measured skewness. All of these changing surface statistics indicate that the contacting asperities become flatter due to the applied load, and that some atoms are displaced to fill parts of the valleys. Hence, the RMS roughness changes only mildly, but the slopes and skewness are much more affected by this displacement effect. We have also directly verified that atoms are displaced by visualizing their trajectories. The observation that the RMS slope changes is important, because it is known that the mean pressure is a function of the RMS slope [14]. Thus, if the sample would be subject to repetitive loading and unloading, leading to local changes in the RMS slope, the mean pressures recorded during each new loading phase would be different. Furthermore, it is already known from simulations, where

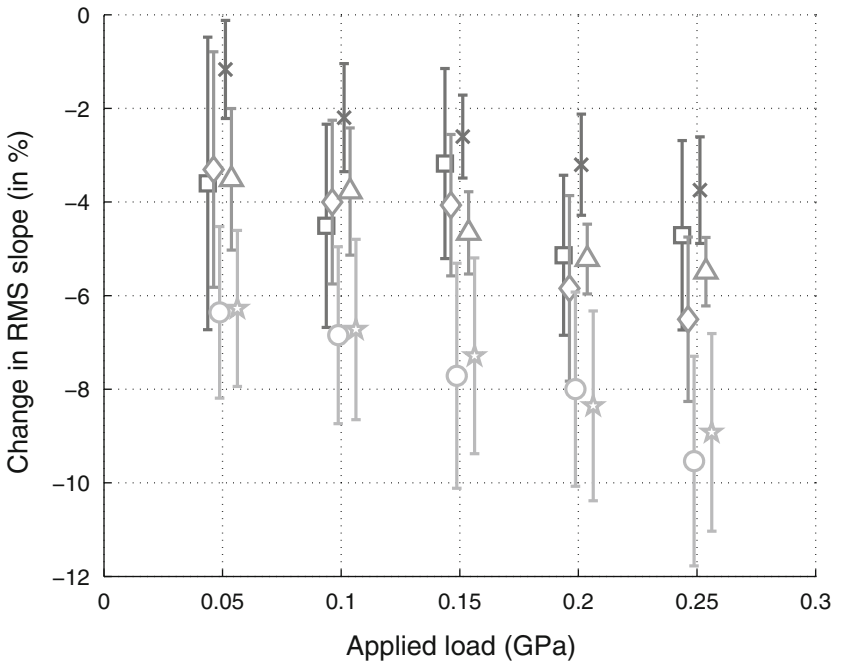

(b) RMS slope

and markers indicate different RMS roughness and adhesive properties. The same legend is used for both figures, but is only shown in a. The minor $x$-offsets for each pressure are introduced for clarity

one of the two surfaces is considered rigid, that a changing surface roughness affects the friction $[7,28]$.

During the simulation the pressure contribution for each atom in contact is computed and in Fig. 9 the probability distributions of these atomic pressures are shown. In this figure markers are used to indicate the different adhesive and surface properties. Furthermore, the average distributions for the two adhesive cases are depicted by solid lines.

From the pressure distributions it can be seen that lower roughnesses give lower compressive, but higher tensile pressure probabilities. In the cases with weak adhesion, compressive pressures are more likely to occur, whereas tensile pressures are more prone to be found with stronger adhesion between the two bodies. Finally, we observe that the adhesive properties influence the shape of the pressure distributions considerably.

\section{Discussion}

In the previous section we have discussed the results that could be directly inferred from our MD simulations. However, closer examination reveals some more intriguing points, which are in need for a more thorough discussion.

For instance, the dependence of contact area on surface roughness as shown in Fig. 7 seems to break down when the RMS roughness increases above $0.5 \mathrm{~nm}$. Apparently, for these high roughnesses the presence of more prominent asperities (the points which will be load bearing) is more important than the actual RMS roughness. 


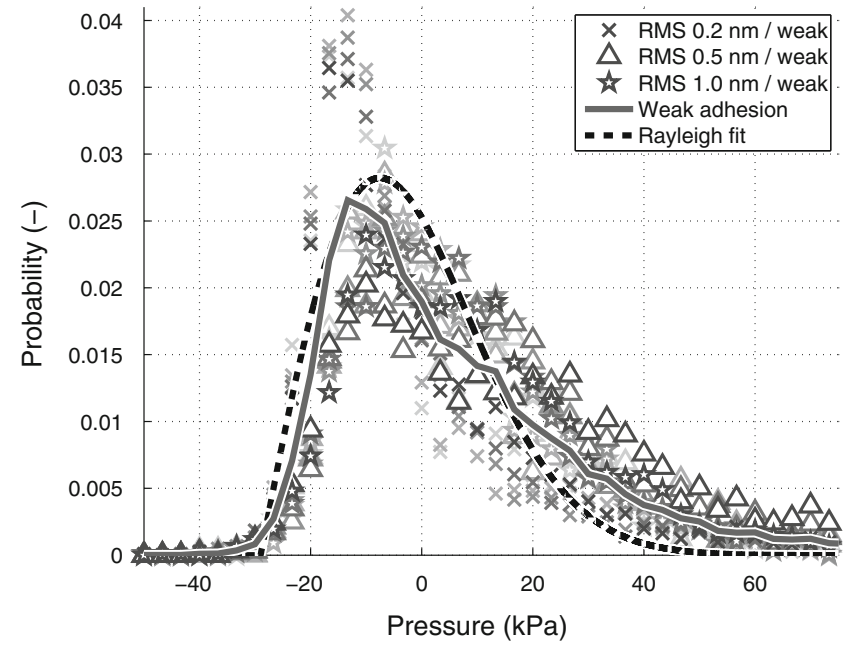

(a) Weak adhesion

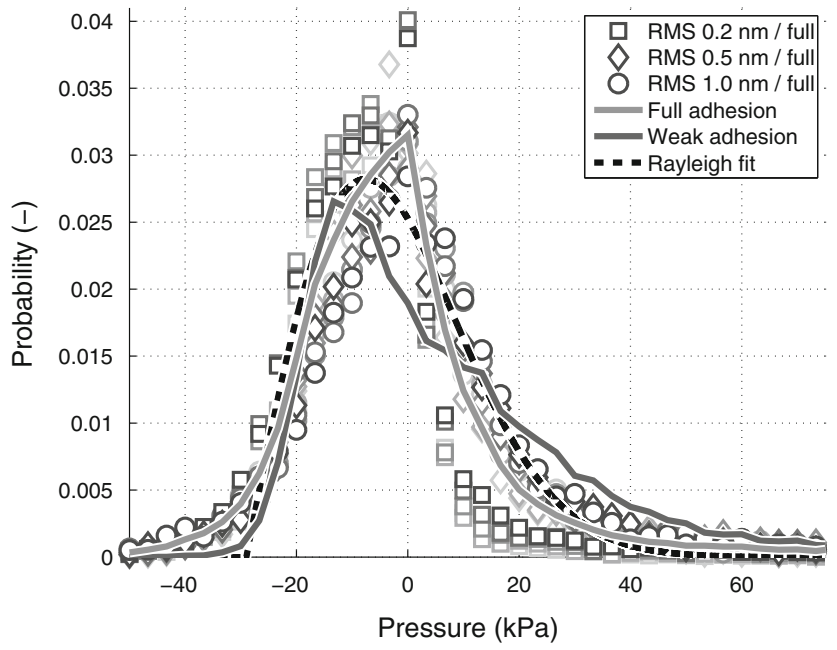

(b) Full adhesion

Fig. 9 Pressure distributions for all atoms in contact in all systems for a weak adhesion and $\mathbf{b}$ full adhesion. The solid lines indicate the average values and negative pressures indicate tension and positive pressures compression. The dotted line in both figures indicates the best fit for a shifted Rayleigh distribution

As pointed out before for the weaker adhesion case, the load-area dependence for the two highest RMS roughnesses seems reversed, which might be explained by the relative small system size $(13 \times 13 \mathrm{~nm}$ in lateral directions $)$. A small system size limits the number of atoms that can be used to model (i.e., discretize) a surface, and as stated before, the presence of prominent asperities becomes more important for a higher RMS roughness. This also means that the pressure being carried by each of these asperities must increase as well (when applying the same load as for lower RMS roughness). This implies that the occurrence of plastic events (e.g., displacing of atoms) is much more likely to occur, which changes the roughness and complicates observing the dependence between contact area and RMS roughness. Yet an additional effect to explain why there is almost no difference in contact area for the two higher RMS roughnesses for this small system could be that, as has been shown by others, that stress distributions of contact clusters change considerably when the system size decreases [7].

Turning our attention to the change in surface characteristics it is noted that although the surface roughness decreased when a load is applied to the body, the change in RMS roughness was minor (around $1 \%$ maximal). However, the standard deviations observed for each applied load are relatively large. These large error bars are also seen for the change in skewness and RMS slope. Because the error bars indicate the differences between the different random seeds used in the rough surface generation, it is clear that the topography (location and size of peaks and valleys) of each of the rough surfaces has a strong effect on the change in roughness due to the applied load. Or, in other words, the different surfaces cannot be characterized only by their statistics as provided in
Table 1, but the topography of the actual surface is important as well. Of course, that there is such a strong dependence on the surface topography is also due to the fact that our systems are relatively small (as has been mentioned above). If we would increase the system size it is very likely that the dependence on topography would decrease. However, increasing the system size increases the computational cost as well. To investigate a sufficiently large number of different roughnesses, adhesive properties and applied loads then becomes prohibitively expensive.

When introducing the contact detection algorithm it was mentioned that our approach allows for the computation of the local pressure on each of the contact clusters. Investigating the relation between the size of the contact clusters and the measured pressures showed that especially for small contact clusters the pressures can vary orders of magnitude. Moreover, there is hardly any difference between different roughnesses, adhesive properties and applied loads. A possible explanation for these irregular local pressure distributions could be that due to plasticity effects at the contacting asperities the surface becomes more amorphous, and thus could lead to more irregular pressure distributions [18].

As one of the final results we showed the pressure distributions for atoms in contact. Although these distributions resemble the shapes of distributions reported by others [7, $25,26]$, which are shown to be in reasonable agreement with Persson's theory, there are some important differences. In the model of Persson the pressure distributions are described by a double-Gaussian function, or in the limit of low loads by a Rayleigh distribution $[22,37,38]$. In all of these cases adhesion is neglected and the material is considered to be linear elastic, and, consequently, the distributions are only defined 
on the positive interval. In our simulations (with adhesion and plasticity) we do observe negative (tensile) pressures, and, thus, our pressure distributions are shifted into the negative interval as well. In Fig. 9 we have added a best fitting 'Rayleigh' distribution (dashed line and shifted into the negative pressure range, because theoretically, a Rayleigh distribution is only defined on the positive interval). From the shapes of the pressure distributions, it can be easily seen that neither matches perfectly the Rayleigh distributions. However, the salient point of our results is that due to the presence of the tensile pressures, the distributions proposed by Persson do not seem to apply for the systems under consideration in this paper.

\section{Conclusion}

Understanding how two surfaces adhere to each other at the atomistic level is key to better understand the origins of friction. To that end we presented the results from MD simulations of two aluminium bodies in contact, where both contact surfaces are considered to have a self-affine fractal roughness.

Furthermore, we implemented a parallel contact detection algorithm within the MD code used for our simulations (LAMMPS), and we discussed the current possibilities of this algorithm, although not all of the features are relevant for a system under normal loading.

In agreement with theoretical models, we have shown that the contact area increases in proportion to normal load, and that, for a fixed load, increasing surface roughness decreases the contact area. Furthermore, we have demonstrated that due to the normal loading the surface roughness changes, the primary mechanism being a flattening of the contacting asperities. As such, the fractal properties of the surface are lost. Finally, it has been shown that the pressure distributions obtained from systems, which do not necessarily behave elastically and which include adhesion between bodies, differ significantly from Greenwood's and Persson's theories.

Acknowledgments The research described in this paper is supported by the European Research Council (ERCstg UFO-240332).

\section{References}

1. Allen MP, Tildesley DJ (1987) Computer Simulation of Liquids. 1st edn. Oxford University Press, Oxford

2. Amontons G (1699) De la resistance causée dans les machines. Mem Acad R A 12:275-282

3. Anciaux G, Molinari JF (2009) Contact mechanics at the nanoscale, a 3D multiscale approach. Int J Numer Meth Eng 79: 1041-1067
4. Archard JF (1953) Contact and rubbing of flat surfaces. J Appl Phys 24:981-988

5. Blau PJ (2001) The significance and use of the friction coefficient. Tribol Int 34:585-591

6. Bowden FP, Tabor D (2001) The friction and lubrication of solids. Oxford University Press, USA

7. Campañà C, Müser MH, Robbins MO (2008) Elastic contact between self-affine surfaces: comparison of numerical stress and contact correlation functions with analytic predictions. J Phys Condens Matter 20:354013

8. Cheng S, Luan B, Robbins MO (2010) Contact and friction of nanoasperities: effects of adsorbed monolayers. Phys Rev E 81(1):016102

9. Ciavarella M, Greenwood J, Paggi M (2008) Inclusion of "interaction" in the Greenwood and Williamson contact theory. Wear 265:729-734

10. Cormen TH, Leiserson CE, Rivest RL, Stein C (2009) Introduction to Algorithms, 3rd edn. MIT Press, Cambridge

11. Coulomb CA (1821) Théorie des machines simples. France, Paris

12. Daw MS, Baskes MI (1984) Embedded-atom method: derivation and application to impurities, surfaces, and other defects in metals. Phys Rev B 29:6443-6453

13. Greenwood JA, Williamson JBP (1966) Contact of nominally flat surfaces. Proc R Soc Lond A 295:300-319

14. Hyun S, Pei L, Molinari JF, Robbins MO (2004) Finite-element analysis of contact between elastic self-affine surfaces. Phys Rev E 70:026117

15. Kale L, Skeel R, Bhandarkar M, Brunner R, Gursoy A, Krawetz N, Phillips J, Shinozaki A, Varadarajan K, Schulten K (1999) NAMD2: greater scalability for parallel molecular dynamics. J Comp Phys 151:283-312

16. Knecht V, Marrink SJ (2007) Molecular dynamics simulations of lipid vesicle fusion in atomic detail. Biophys J 92:4254-4261

17. Lennard-Jones JE (1931) Cohesion. Proc Phys Soc 43:461-482

18. Luan B, Robbins MO (2005) The breakdown of continuum models for mechanical contacts. Nature 435:929-932

19. Luan B, Robbins MO (2006) Contact of single asperities with varying adhesion: comparing continuum mechanics to atomistic simulations. Phys Rev E 74:026111

20. Luan B, Robbins MO (2009) Hybrid atomistic/continuum study of contact and friction between rough solids. Tribol Lett 36:1-16

21. Mandelbrot BB, Passoja DE, Paullay AJ (1984) Fractal character of fracture surfaces of metals. Nature 308:721-722

22. Manners W, Greenwood J (2006) Some observations on Persson's diffusion theory of elastic contact. Wear 261:600-610

23. Markvoort AJ, Pieterse K, Steijaert MN, Spijker P, Hilbers PAJ (2005) The bilayer-vesicle transition is entropy-driven. J Phys Chem B 109:22649-22654

24. Miller GSP (1986) The definition and rendering of terrain maps. SIGGRAPH '86: Proceedings of the 13th annual conference on Computer graphics and interactive techniques. ACM, New York, pp 39-48

25. Mo Y, Szlufarska I (2010) Roughness picture of friction in dry nanoscale contacts. Phys Rev B 81:035405

26. Mo Y, Turner KT, Szlufarska I (2009) Friction laws at the nanoscale. Nature 457:1116-1119

27. Morse PM (1929) Diatomic molecules according to the wave mechanics. II. Vibrational levels. Phys Rev 34:57-64

28. Mosey NJ, Müser MH (2007) Atomistic modeling of friction. Rev Comp Chem 25:67-124

29. Nye JF (1985) Physical properties of crystals. Oxford University Press, Oxford

30. Paggi M, Ciavarella M (2010) The coefficient of proportionality $\kappa$ between real contact area and load, with new asperity models. Wear 268:1020-1029 
31. Persson BNJ, Albohr O, Tartaglino U, Volokitin AI, Tosatti E (2005) On the nature of surface roughness with application to contact mechanics, sealing, rubber friction and adhesion. J Phys Condens Matter 17:R1-R62

32. Plimpton S (1995) Fast parallel algorithms for short-range molecular dynamics. J Comp Phys 117:1-19

33. Spijker P, van Hoof B, Debertrand M, Markvoort AJ, Vaidehi N, Hilbers PAJ (2010) Coarse grained molecular dynamic simulations of transmembrane protein-lipid systems. Int J Mol Sci 11: 2393-2420

34. Spijker P, Markvoort AJ, Nedea SV, Hilbers PAJ (2010) Computation of accommodation coefficients and the use of velocity correlation profiles in molecular dynamics simulations. Phys Rev E 81:011203
35. Tadmor E, Ortiz M, Phillips R (1996) Quasicontinuum analysis of defects in solids. Philos Mag A Phys Cond Mat 73:1529-1563

36. Voss RF (1985) Fundamental algorithms in computer graphics. Springer, Berlin

37. Yang C, Persson BNJ (2008) Contact mechanics: contact area and interfacial separation from small contact to full contact. J Phys Condens Matter 20:215214

38. Yang C, Persson BNJ (2008) Molecular dynamics study of contact mechanics: contact area and interfacial separation from small to full contact. Phys Rev Lett 100:024303

39. Yang C, Tartaglino U, Persson BN (2006) A multiscale molecular dynamics approach to contact mechanics. Eur Phys J E 19:47-58 